International Journal of Pure and Applied Mathematics

Volume 112 No. $3 \quad 2017,649-653$

ISSN: 1311-8080 (printed version); ISSN: 1314-3395 (on-line version)

url: http://www.ijpam.eu

doi: 10.12732 /ijpam.v112i3.16

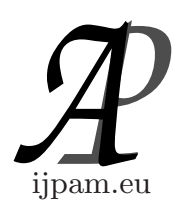

\title{
SUPERCYCLICITY OF TUPLES IN THE HILBERT-SCHMIDT OPERATORS
}

\author{
B. Yousefi ${ }^{1 \S}$, A. Sharifi ${ }^{2}$ \\ ${ }^{1,2}$ Department of Mathematics \\ Payame Noor University \\ P.O. Box 19395-4697, Tehran, IRAN
}

\begin{abstract}
In this paper, we investigate the supercylicity for any tuple of operators in the class of Hilbert Schmidt operators. Also, the same result for hypercyclicity of tuples of operators is considered.
\end{abstract}

AMS Subject Classification: 47B37, 47B33

Key Words: Hilbert-Schmidt operator, tuple of operators, supercyclic vector, hypercyclic operator, topologically transitive

\section{Introduction}

Let $\mathcal{T}=\left(T_{1}, T_{2}, \ldots, T_{n}\right)$ be an n-tuple of commuting operators acting on an infinite dimensional Banach space $X$. We will let $\mathcal{F}=\left\{T_{1}{ }^{k_{1}} T_{2}{ }^{k_{2}}, \ldots, T_{n}{ }^{k_{n}}: k_{i} \in\right.$ $\left.\mathbf{Z}_{+}, \quad i=1, \ldots, n\right\}$ be the semigroup generated by $\mathcal{T}$. For $x \in X$, the orbit of $x$ under the tuple $\mathcal{T}$ is the set $\operatorname{Orb}(\mathcal{T}, x)=\{S x: S \in \mathcal{F}\}$. A vector $x$ is called a hypercyclic vector for $\mathcal{T}$ if $\operatorname{Orb}(\mathcal{T}, x)$ is dense in $X$ and in this case the tuple $\mathcal{T}$ is called hypercyclic. Also, a vector $x$ is called a supercyclic vector for $\mathcal{T}$ if $\operatorname{COrb}(\mathcal{T}, x)$ is dense in $X$ and in this case the tuple $\mathcal{T}$ is called supercyclic. We say that a tuple $\mathcal{T}=\left(T_{1}, T_{2}, \ldots, T_{n}\right)$ is topologically transitive with respect to a tuple of nonnegative integer sequences $\left(\left\{k_{j(1)}\right\}_{j},\left\{k_{j(2)}\right\}_{j}, \ldots,\left\{k_{j(n)}\right\}_{j}\right)$, if

\footnotetext{
Received: $\quad$ January 6, 2017

Revised: January 31, 2017

Published: February 9, 2017

(c) 2017 Academic Publications, Ltd.

url: www.acadpubl.eu

$\S_{\text {Correspondence author }}$
} 
for every nonempty open subsets $U, V$ of $X$ there exists $j_{0} \in \mathbb{N}$ such that $T_{1}^{k_{j_{0}(1)}} T_{2}^{k_{j_{0}(2)}} \ldots T_{n}^{k_{j_{0}(n)}}(U) \cap V \neq \varnothing$. Also, we say that an n-tuple $\mathcal{T}$ is topologically transitive if it is topologically transitive with respect an n-tuple of nonnegative integer sequences. If $X$ is separable, topologically transitivity of $\mathcal{T}$ implies the hypercyclicity of $\mathcal{T}$. For several works see [1-3].

We begin with the following well known theorems that are the main tools we use to show that a tuple of operators is supercyclic.

Theorem 1.1. (Supercyclicity Criterion for Tuples of Operators) Let $\mathcal{T}=\left(T_{1}, T_{2}, \ldots, T_{n}\right)$ be an n-tuple of operators acting on an infinite dimensional Banach space $X$. The tuple $\mathcal{T}$ is supercyclic whenever there exist two dense subsets $Y$ and $Z$ in $X$, and strictly increasing sequences $\left\{m_{j_{i}}\right\}_{j}$ for $i=1, \ldots, n$, and a sequence of mappings $S_{j}: Z \rightarrow X$ such that:

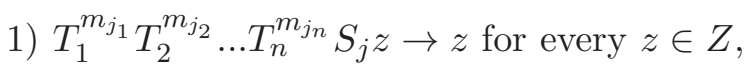

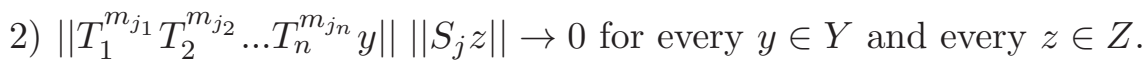

Theorem 1.2. Let $X$ be a separable infinite dimensional Banach space and $\mathcal{T}=\left(T_{1}, T_{2}, \ldots, T_{n}\right)$ be a tuple of operators $T_{1}, T_{2}, \ldots, T_{n}$. Then $\mathcal{T}$ is supercyclic, if and only if for any two nonempty open sets $U$ and $V$, there exist $m_{i} \geq 1$ for $i=1, \ldots, n$ and $\lambda \in \mathbf{C} \backslash\{0\}$ such that $\lambda T_{1}^{m_{1}} \ldots T_{n}^{m_{n}}(U) \cap V \neq \varnothing$.

\section{Main Results}

We will give necessary and sufficient conditions for the supercyclicity of a tuple in the norm of Hilbert-Schmidt operators. Recall that if $\left\{e_{i}\right\}$ is a basis for a separable Hilbert space $H, A \in B(H)$, and $\|A\|_{2}<\infty$, then $\mathrm{A}$ is called a Hilbert-Schmidt operator. The set of Hilbert-Schmidt operators on $H$ is denoted by $B_{2}(H)$. We note that $B_{2}(H)$ with \|\|$_{2}$-topology is separable. For this suppose that $\left\{e_{i}\right\}$ is the basis for the separable Hilbert space $H$, also suppose that $S$ is a dense subset in $H$. Then $S(H)$ will denote the set of all finite rank operators $E$ such that there exists $N \in \mathbb{N}$ satisfying $E\left(e_{n}\right)=0$ for $n \geq N$ and $E\left(e_{n}\right) \in S$ for $n<N$. Clearly, $S(H)$ is \|\|$_{2}$-dense in $B_{2}(H)$ and if $S$ is countable, then $S(H)$ is also countable. For any operator $T \in B(H)$, the left multiplication operator $L_{T}: B(H) \rightarrow B(H)$ is defined by $L_{T}(S)=T S$ for all $S$ in $B(H)$. If $\mathcal{T}=\left(T_{1}, T_{2}, \ldots, T_{n}\right)$ is an n-tuple of operators acting on $H$, then by $\mathcal{L}_{T_{1}, \ldots, T_{n}}$ we mean the tuple $\left(L_{T_{1}}, L_{T_{2}}, \ldots, L_{T_{n}}\right)$. Recall that for vectors $g, h$ in $H$ the operator $g \otimes h$ denotes a rank one operator and is defined by $(g \otimes h)(f)=<f, h>g$. 
Theorem 2.1. Let $\mathcal{T}=\left(T_{1}, T_{2}, \ldots, T_{n}\right)$ be an n-tuple of operators acting on $H$. The following are equivalent:

i) For each pair $U, V$ of nonempty open subsets of $H$, and each neighborhood $W$ of zero, $\lambda T_{1}{ }^{k_{1}} T_{2}{ }^{k_{2}} \ldots T_{n}{ }^{k_{n}} U \cap W \neq \phi$ and $\lambda T_{1}{ }^{k_{1}} T_{2}{ }^{k_{2}} \ldots T_{n}{ }^{k_{n}} W \cap V \neq \phi$ for some integers $k_{i} \geq 1, i=1, \ldots, n$, and $\lambda \in \mathbf{C} \backslash\{0\}$.

ii) $\mathcal{L}_{T_{1}, \ldots, T_{n}}=\left(L_{T_{1}}, L_{T_{2}}, \ldots, L_{T_{n}}\right)$ is $\|\cdot\|_{2}$-supercyclic on $B_{2}(H)$.

Proof. (i) implies (ii): Suppose that $G, Q$ are nonempty $\|.\|_{2}$-open subsets of $B_{2}(H)$. We will show that there exist a $\lambda \in \mathbb{C} \backslash\{0\}$ and some integers $m_{i} \geq 1, i=1, \ldots, n$ such that $U^{\prime} \cap \lambda L_{T_{1}}^{-m_{1}} L_{T_{2}}^{-m_{2}} \ldots L_{T_{n}}^{-m_{n}} V^{\prime} \neq \phi$. For this fix an orthonormal basis $E=\left\{e_{i}: i \geq 1\right\}$ for $H$ and choose finite rank operators $A$ and $B$ such that $A \in S(H) \cap G$ and $B \in S(H) \cap Q$. Hence for certain integer $N \geq 1$ we have $A e_{i}=B e_{i}=0$ for $i>N$. But for some $\varepsilon>0$ we have $\left\{S \in S(H):\|S-A\|_{2}<\varepsilon\right\} \subseteq G,\left\{S \in S(H):\|S-B\|_{2}<\varepsilon\right\} \subseteq Q$. Now consider the open sets

$$
U_{i}=\left\{h \in H:\left\|h-A e_{i}\right\|<\frac{\varepsilon}{2 \sqrt{N}}\right\}, V_{i}=\left\{h \in H:\left\|h-B e_{i}\right\|<\frac{\varepsilon}{2 \sqrt{N}}\right\}
$$

for $i=1,2, \cdots, N$. Now by Theorem 1.2 , it follows that there exist integers $k_{i, j}, r_{i, j}: i=1, \ldots, N-1, j=1, \ldots, n$ and scalars $\lambda_{i}, \beta_{i}, i=1, \ldots, N-1$, in $\mathbf{C} \backslash\{0\}$ such that

$$
\begin{gathered}
U=U_{1} \cap \lambda_{1} T_{1}^{-k_{1,1}} \ldots T_{n}^{-k_{1, n}} U_{2} \cap \cdots \cap \lambda_{N-1} T_{1}^{-k_{N-1,1}} \ldots T_{n}^{-k_{N-1, n}} U_{N} \neq \phi, \\
V=V_{1} \cap \beta_{1} T_{1}^{-r_{1,1}} \ldots T_{n}^{-r_{1, n}} V_{2} \cap \cdots \cap \beta_{N-1} T_{1}^{-r_{N-1,1} \ldots T_{n}{ }^{-r_{N-1, n}} V_{N} \neq \phi .}
\end{gathered}
$$

Put $k_{0, j}, r_{0, j}=0$ for all $j=1, \ldots, n$ and let $W=\{h:\|h\|<\delta\}$ where $\delta=$ $\min \left\{\delta_{i}: 1 \leq i \leq N\right\}$ and

$$
\delta_{i}=\frac{1}{2 \sqrt{N}} \min \left\{\frac{\varepsilon\left|\lambda_{i}\right|}{\left\|T_{1}\right\|^{k_{i-1,1}} \ldots|| T_{n} \|^{k_{i-1, n}}}, \frac{\varepsilon\left|\beta_{i}\right|}{\left.\left\|T_{1}||^{r_{i-1,1} \ldots .}|| T_{n}\right\|\right|^{r_{i-1, n}}}\right\} .
$$

Since $T$ satisfies the hypothesis (i) of the Theorem, there exists some $\lambda \in$ $\mathbf{C} \backslash\{\},. x \in W$ and $y \in U$ such that

$$
\lambda T_{1}^{m_{1}} T_{2}{ }^{m_{2}} \ldots T_{n}{ }^{m_{n}} x \in V ; \lambda T_{1}^{m_{1}} T_{2}^{m_{2}} \ldots T_{n}^{m_{n}} y \in W
$$

for some integers $m_{i}: i=1, \ldots, n$. The above relations imply that

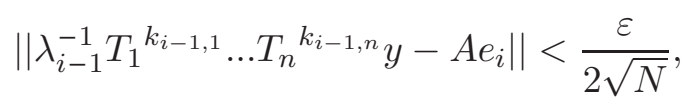




$$
\left\|T_{1}^{m_{1}} \ldots T_{n}^{m_{n}} \lambda\left(\beta_{i}^{-1} T_{1}^{r_{i-1,1}} \ldots T_{n}^{r_{i-1, n}} x\right)-B e_{i}\right\|<\frac{\varepsilon}{2 \sqrt{N}}
$$

for $i=1,2, \cdots, N$. Note that $T_{1}^{k_{0,1}} T_{2}{ }^{k_{0,2}} \ldots T_{n}{ }^{k_{0, n}} y=y$ and $T_{1}^{r_{0,1}} T_{2}{ }^{r_{0,2}} \ldots T_{n}{ }^{r_{0, n}} x=$ $x$. Now define $S_{1}$ by $S_{1}=\sum_{i=1}^{N} \lambda_{i}^{-1} T_{1}^{k_{i-1,1}} T_{2}{ }^{k_{i-1,2}} \ldots T_{n}{ }^{k_{i-1, n}} y \otimes e_{i}$ and $S_{2}$ by

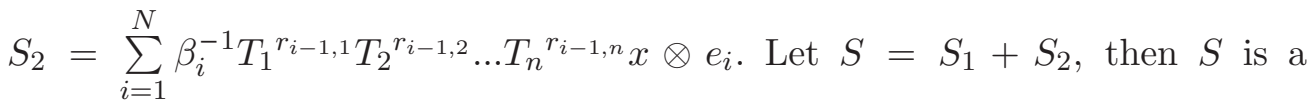
Hilbert-Schmidt operator, because it has finite rank. Note that since $x \in W$, by definition of $\delta$, we have

$$
\left\|\beta_{i}^{-1} T_{1}^{r_{i-1,1}} T_{2}^{r_{i-1,2}} \ldots T_{n}^{r_{i-1, n}} x\right\|<\frac{\varepsilon}{2 \sqrt{N}} .
$$

Now we get the following inequality:

$$
\begin{aligned}
\|S-A\|_{2} \leq\left\|S_{1}-A\right\|_{2}+\left\|S_{2}\right\|_{2} & =\left\{\sum_{i=1}^{N} \| \lambda_{i}^{-1} T_{1}^{k_{i-1,1}} \ldots T_{n}^{\left.{ }^{k_{i-1, n}} y-A e_{i} \|^{2}\right\}^{\frac{1}{2}}}\right. \\
& +\left\{\sum_{i=1}^{N}\left\|\beta_{i}^{-1} T_{1}^{r_{i-1,1}} \ldots T_{n}^{r_{i-1, n}} x\right\|^{2}\right\}^{\frac{1}{2}}<\varepsilon
\end{aligned}
$$

Hence $S \in G$. Also since $\lambda T_{1}^{m_{1}} T_{2}^{m_{2}} \ldots T_{n}^{m_{n}} y \in W$, we have

$$
\left\|\lambda \lambda_{i}^{-1} T_{1}^{k_{i-1,1}} T_{2}^{k_{i-1,2}} \ldots T_{n}^{k_{i-1, n}}\left(T_{1}^{m_{1}} T_{2}^{m_{2}} \ldots T_{n}^{m_{n}} y\right)\right\|<\frac{\varepsilon}{2 \sqrt{N}} .
$$

Thus we obtain

$$
\begin{aligned}
\left\|\lambda L_{T_{1}}^{m_{1}} \quad L_{T_{2}}^{m_{2}} \quad \ldots L_{T_{n}}^{m_{n}} S-B\right\|_{2} \leq\left\|\lambda L_{T_{1}}^{m_{1}} L_{T_{2}}^{m_{2}} \ldots L_{T_{n}}^{m_{n}} S_{2}-B\right\|_{2} \\
\quad+\quad\left\|\lambda L_{T_{1}}^{m_{1}} L_{T_{2}}^{m_{2}} \ldots L_{T_{n}}^{m_{n}} S_{1}\right\|_{2} \\
\quad=\quad\left\{\sum_{i=1}^{N}\left\|\lambda T_{1}^{m_{1}} T_{2}^{m_{2}} \ldots T_{n}^{m_{n}}\left(\beta_{i}^{-1} T_{1}^{r_{i-1,1}} \ldots T_{n}^{r_{i-1, n}} x\right)-B e_{i}\right\|^{2}\right\}^{\frac{1}{2}} \\
\quad \\
\quad\left\{\sum_{i=1}^{N}\left\|\lambda \lambda_{i}^{-1} T_{1}^{k_{i-1,1}} T_{2}^{k_{i-1,2}} \ldots T_{n}^{k_{i-1, n}}\left(T_{1}^{m_{1}} T_{2}^{m_{2}} \ldots T_{n}^{m_{n}} y\right)\right\|^{2}\right\}^{\frac{1}{2}}<\varepsilon .
\end{aligned}
$$

So $\lambda L_{T_{1}}^{m_{1}} L_{T_{2}}^{m_{2}} \ldots L_{T_{n}}^{m_{n}} S \in Q$. Now it follows that $G \cap \lambda L_{T_{1}}^{-m_{1}} L_{T_{2}}^{-m_{2}} \ldots L_{T_{n}}^{-m_{n}} Q \neq \phi$ and so by Theorem 1.2, $\mathcal{L}_{T_{1}, \ldots, T_{n}}$ is \|\|$_{2}$-supercyclic. (ii) implies (i): If (i) holds, then it is well known that $\mathcal{T}$ satisfies the supercyclicity criterion and this completes the proof. 
Now we state the hypercyclicity criterion for tuples of operators.

Theorem 2.2. (Hypercyclicity Criterion for tuples of operators) Suppose that $X$ is a separable infinite dimensional Banach space and $\mathcal{T}=\left(T_{1}, T_{2}, \ldots, T_{n}\right)$ be the n-tuple of operators $T_{1}, T_{2}, \ldots, T_{n}$ acting on $X$. If there exist two dense subsets $Y$ and $Z$ in $X$, and strictly increasing sequences $\left\{m_{j}(i)\right\}_{j}$ for $i=1, \ldots, n$ such that:

1. $T_{1}^{m_{j}(1)} \ldots T_{n}^{m_{j}(n)} y \rightarrow 0$ for all $y \in Y$,

2. there exist a sequence of functions $\left\{S_{j}: Z \rightarrow X\right\}$ such that for every $z \in Z, S_{j} z \rightarrow 0$, and $T_{1}^{m_{j}(1)} \ldots T_{n}^{m_{j}(n)} S_{j} z \rightarrow z$ as $j \rightarrow \infty$.

Then $\mathcal{T}$ is a hypercyclic tuple.

Remark 2.3. In the proof of Theorem 2.1, the supercyclicity criterion for tuples has an important rule. Similarly, by using Theorem 2.2 and the method of three open sets $U, V$ and $W$ used in Theorem 2.1, we can obtain the same result for $\|.\|_{2}$-hypercyclicity of tuples on $B_{2}(H)$, so we omit the proof.

\section{References}

[1] N.S. Feldman, Hypercyclic tuples of operators and somewhere dense orbits, J. Math. Appl., 346 (2008), 82-98.

[2] B.F. Madore and R.A. Martinez-Avendano, Subsapce hypercyclicity, Journal of Mathematical Analysis and Applications, 375, No. 2 (2011), 502-511.

[3] B. Yousefi, Hereditarily transitive tuples, Rend. Circ. Mat. Palermo, Volume 2011, doi: 10.1007/S12215-011-0066-y. 
